\title{
Intuitive procedure for constructing geometrically complex objects using cyclides
}

\author{
Y L Srinivas and Debasish Dutta
}

\begin{abstract}
In the past, cyclide surfaces have been used effectively for the variable radius blending of natural quadric intersections. However, attempts to use cyclides for constructing realistic, freeform composite surfaces met with rather limited success. The paper presents a simple procedure for creating and manipulating geometrically complex objects using tubular cyclide pieces. The method described is intuitive from the designer's point of view, and it is based on the fundamental definitions and properties of the cyclide. Various practical issues involved in this design procedure are discussed, several extensions of the basic technique are described, and implemented examples are provided.
\end{abstract}

Keywords: Dupin cyclides, surface synthesis, solid modelling

Cyclides were discovered in 1822 by Dupin, a French mathematician ${ }^{1}$. He defined a cyclide as follows:

Definition: A cyclide is the envelope of all variable radius spheres that are tangent to three fixed spheres.

Cyclide surfaces received considerable attention from classical mathematicians of the late 19 th century. Some of the most noted works on these surfaces were written by Maxwell $^{2}$ and Cayley ${ }^{3}$. However, interest in these surfaces tapered off gradually, resulting in their omission from many classical geometry textbooks. In the early 1980 s, these surfaces were rediscovered for use in computer-aided geometric design (CAGD), mainly for surface composition using principal patches ${ }^{4}$. Subsequent investigation of the properties of cyclides for use in surface modelling ${ }^{5-7}$ revealed that these surfaces have a strong

Department of Mechanical Engineering, University of Michigan, Ann Arbor, MI 48105, USA

Paper received: 31 May 1993. Revised: 15 October 1993 potential for applications in geometric design. In particular, the cyclide might be a good addition to the set of existing constructive solid geometry (CSG) primitives (sphere, cone, cylinder, torus), with potential benefits such as enhanced geometric flexibility and a broader application domain. Additionally, all existing primitives are special cases of cyclides - a fact that can potentially lead to a unified computational framework. More importantly, owing to the enhanced geometric flexibility afforded by cyclides, a certain degree of freeform-like object modelling can be achieved in the CSG domain for the first time.

The use of cyclide patches for freeform surface design was initiated about a decade ago. A principal-patch formulation for the cyclide surface was given by Martin ${ }^{4}$. De Pont ${ }^{6}$ outlined several different versions of this formulation, and used them for surface intersections. He also explored the possibility of putting these patches together to form composite surfaces. Sharrock ${ }^{8}$ studied the patch composition problem in depth, and developed techniques for surface design using cyclides. McLean ${ }^{9}$ described a different approach to patch composition using spherical mapping. This method is not tied to a particular formulation of the patch, but is based on the geometry of the patch.

However, the use of cyclide surfaces for freeform surface design has seen few practical applications. One reason, we conjecture, for this lack of acceptance is the nonintuitive nature of the synthesis techniques ${ }^{9,10}$. While many of the formulations referred to above are mathematically compact and elegant, a designer might conceive them to be mathematically complex and somewhat abstract in nature. Most designers are simply more comfortable dealing with concrete quantities such as radii, areas and volumes than with abstract mathematical concepts such as pentaspherical spaces. Consequently, synthesis techniques that are intuitive and 
geometrically less taxing to the mechanical designer will be helpful. This is the motivation for the present work.

\section{SOME RELEVANT PROPERTIES OF CYCLIDES}

Cyclides are 4 th-degree algebraic surfaces (quartics) with several interesting properties, such as circular lines of curvature, closure under offsetting, and closure under inversion. A good treatment of the geometric properties of cyclides can be found in References 11 and 14. For the purpose of the present work, we select a few of these properties for detailed discussion.

Property 1 (fundamental definition of cyclide): A cyclide is the envelope of all spheres that are tangent to three fixed spheres ${ }^{1}$.

From this definition, it follows ${ }^{14}$ that the cyclide surface separates two distinct and nonintersecting families of spheres, which together span the $\mathbb{E}^{3}$ space. It also is evident that a particular cyclide can be uniquely described by specifying three spheres from either of the families. An example is shown in Figure 1, which shows a section of the cyclide on the plane passing through the centres of the three defining spheres. This property is used in the composition procedure described below. We state this property as a corollary for future reference.

Corollary 1: Three distinct, maximal spheres, with the condition that they belong to one family, uniquely determine a cyclide surface, and also the complementary family of spheres.

Property 2: The cyclide possesses two planes of symmetry.

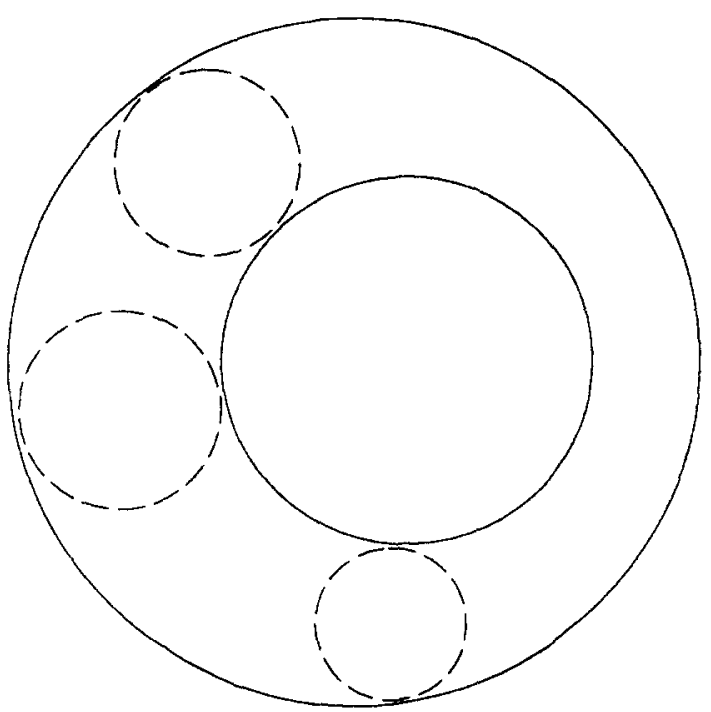

Figure 1 Ring cyclide section on plane through centres of defining spheres
Sectioning the cyclide surface with these planes yields a pair of bounding circles in each plane. Further, the cyclide is uniquely determined by the specification of the bounding circles in either of these planes.

The planes of symmetry of the cyclide are the planes that also contain the spine curves* of the cyclide ${ }^{14}$. The cyclide has two spine curves and each is a conic. The two conics are in the orthogonal planes such that the foci of one coincides with the vertices of the other, and vice versa. Two conics in this spatial configuration are referred to as anticonics. Since a plane of symmetry passes through the centres of all the spheres of one family, these spheres are sectioned into great circles in this plane (see Figure 2). Thus, the bounding circles are the envelopes of a family of circles with their centres on the spine curve. It is evident that the bounding cycles are uniquely determined by specifying three circles from this family. This observation is stated in the following corollary:

Corollary 2: The bounding circles (and the corresponding cyclide) are uniquely determined by specifying three circles in a plane of symmetry, which is equivalent to specifying three spheres belonging to one family.

Property 3: Along a line of curvature on the cyclide, the surface normals form a right circular cone whose apex lies on one of the spine curves.

This property can be explained in the following manner. Consider the cyclide and the family of spheres that are either 'inside' or 'outside'. Each member sphere of this family shares a circle (in general not a great circle) with the cyclide surface. These circles are lines of curvature on the cyclide. At these circles, the collection of surface normals to the cyclide is identical to the collection of surface normals to the sphere. On the sphere, these surface normals form a cone. The apex of the cone is the centre of the sphere which lies on the spine curve of the cyclide.

An interesting observation is that the tangents to the surface along this line of curvature also meet in a cone, which is referred to as the tangent cone of the surface. Hence, it is possible to 'twist' the cyclide about the axis of the tangent cone of a line of curvature without disturbing the $G^{1}$ continuity of the surface along that line of curvature. An example of such an axis of rotation is shown in Figure 3. This observation enables us to generate truly $3 \mathrm{D}$ constructs using cyclide pieces. The following corollary summarizes the above discussion:

Corollary 3: A tangency-preserving rotation can be applied to a piece of the cyclide surface at any line of curvature by twisting the piece about the axis of the tangent cone corresponding to that line of curvature.

*Spine curves refer to the loci of the centres of the spheres that belong to the two families. 

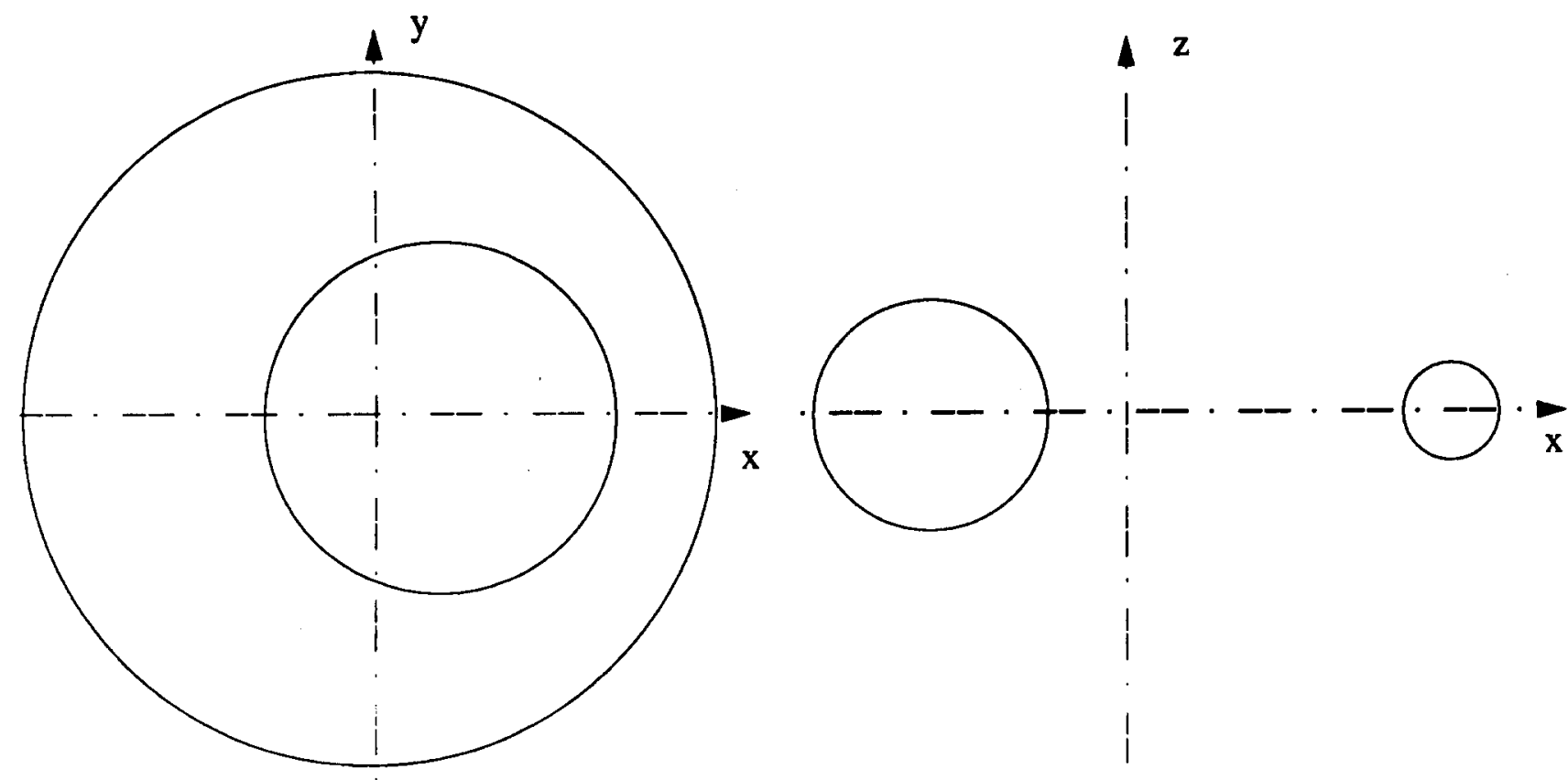

Figure 2 Sections of ring cyclide on two planes of symmetry

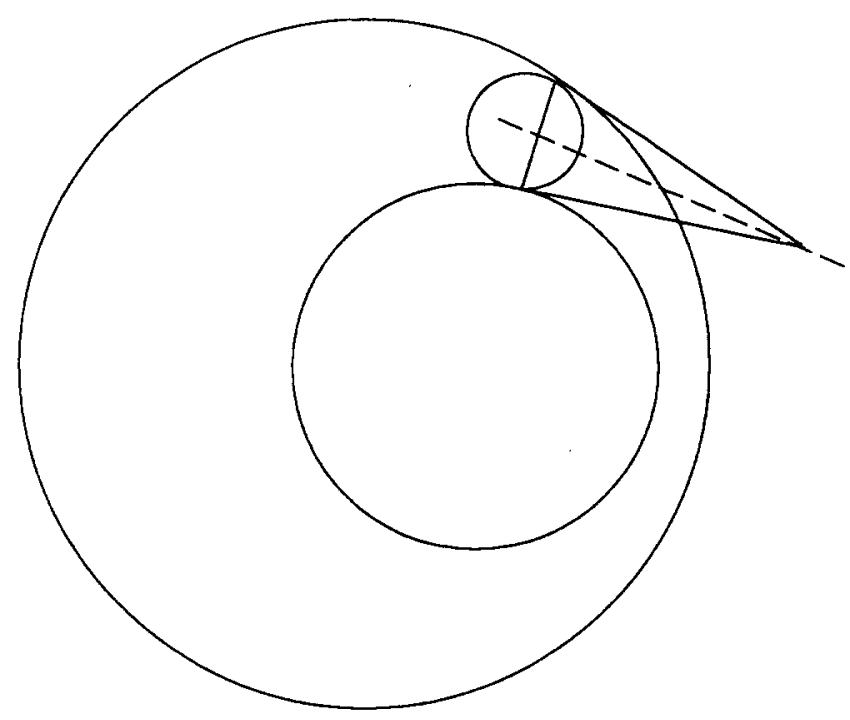

Figure 3 Tangent cone of cyclide at line of curvature

Having discussed these properties and observations, we now proceed to the description of the proposed composition technique. In the following sections, we first discuss the unconstrained circle composition technique (UCCT), followed by the tangency-constrained circle composition technique (TCCT). We then describe two editing methods for local modification of the composite surface. This is followed by a set of implemented examples that illustrate the results obtained using these methods.

\section{UNCONSTRAINED (BASIC) CIRCLE COMPOSITION TECHNIQUE}

A circle composition technique (CCT) was first

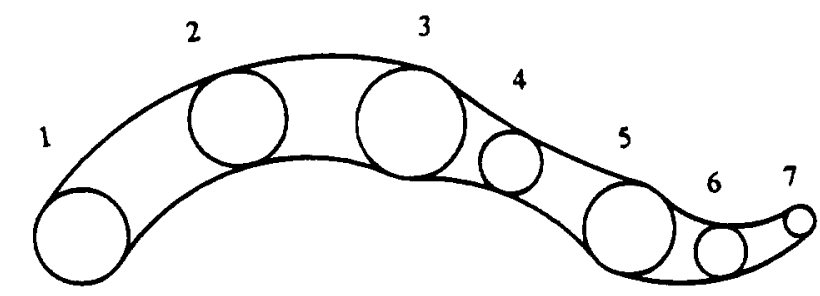

Figure 4 Tubular surface generated by UCCT

developed ${ }^{12}$ for the generation of tubular pipe layouts and motion planning in 3D. The method extends to the present goal of freeform-like object composition in a natural manner. In the UCCT procedure, the object is composed by joining cyclide pieces together. Using Corollary 2 , we can define each cyclide piece using three spheres. However, since we require some degree of connectivity between these pieces, we also require that the $i$ th cyclide piece share a common sphere with the $(i-1)$ th and $(i+1)$ th cyclide piece. In other words, given a series of ordered spheres, we interpolate three of them at a time, in sequence, to obtain a composite volume bounded by cyclide pieces (see Figure 4). This process can be stated in pseudocode form as follows:

\footnotetext{
Procedure UCCT() \{

Circle $c$ [number_of_circles]; /* defining circles */

Cyclide piece [number_of_cyclides]; ${ }^{*}$ cyclide pieces */

int $i=0 ; \quad / *$ circle count $* /$

int count $=0 ; \quad / *$ cyclide count $* /$
} 


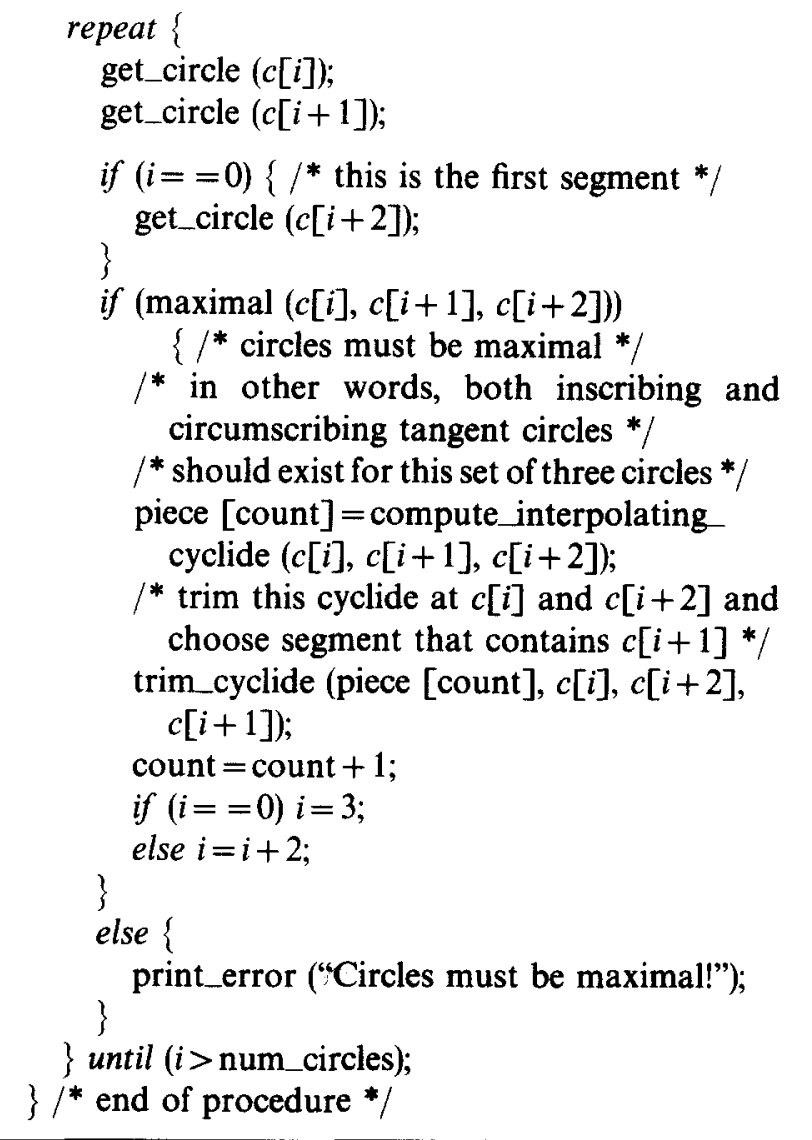

\section{Comments on UCCT}

First, we observe that the centres of the spheres need not be in a single plane. For each piece, the plane of symmetry of the cyclide segment is determined by the centres of the three defining spheres. In the examples presented, we restrict ourselves to the planar case only to maintain visual clarity.

Second, we note that, since each piece is defined uniquely for the corresponding spheres without any constraints, these pieces in general will not match up, resulting in intersections (overlapped cyclide segments) or gaps. For the UCCT, the only way to avoid the intersection of successive segments is to control the location and size of the circles. In the case of gaps between successive segments, the problem can be alleviated by including a part of the surface of the spheres at the junctions. By using this remedy and choosing the location and the size of the circles with some care, one can obtain a connected surface (volume) that is piecewise-tangentcontinuous. This procedure is used to describe the free volume for motion-planning applications ${ }^{12}$, where the mathematical description of the volume is much more critical than the aesthetic appearance of the surface. However, this might not be suitable for applications with stringent shape requirements. At the junctions, the introduction of spherical surfaces results in the 'spherical kneecap' phenomenon ${ }^{13}$, as shown in Figure 5. This

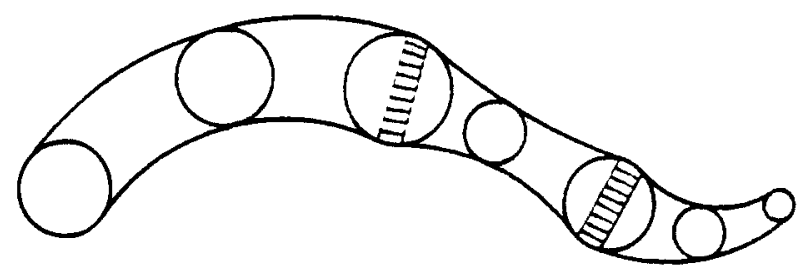

Figure 5 Spherical kneecaps on surface generated by UCCT
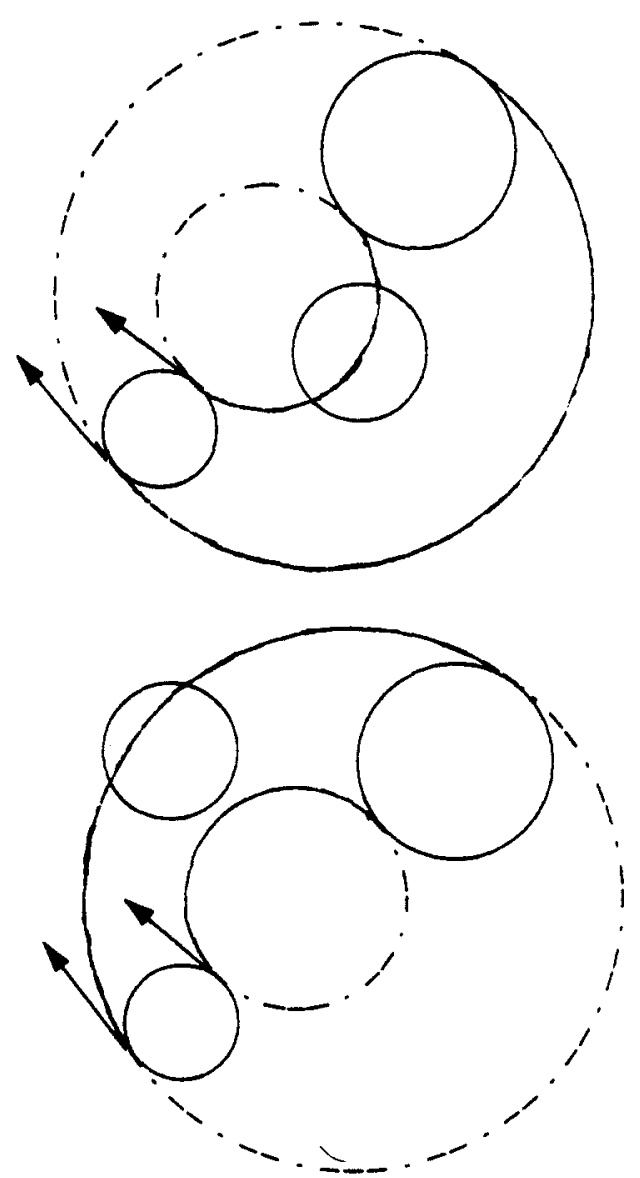

Figure 6 Choice of cyclide surface on basis of junction sphere

results in a sharp change of curvature and sometimes even a change in the sign of the curvature across these junctions. The result is a surface that might not satisfy aesthetic and functional requirements. For a more flexible freeform composition, we need to ensure tangent continuity between these pieces without interposing a spherical surface.

\section{TANGENCY-CONSTRAINID CIRCLE COMPOSITION TECHNIQUE}

In the UCCT, we constructed each cyclide piece without constraints on the adjoining pieces. In the TCCT, we consider the situation in which the $i$ th cyclide piece is required to be tangent-continuous with the $(i-1)$ th piece, without the intermediate spherical surface. Let $c_{j}, c_{j+1}$ 
and $c_{j+2}$ be the circles corresponding to the $i$ th cyclide piece. To construct bounding circles, we note that one point and the tangent at that point are fixed for each of these circles. Since a circle is determined by three constraints, it is not possible to interpolate $c_{j}, c_{j+1}$ and $c_{j+2}$ while satisfying the tangent constraints. Therefore, we choose to interpolate two circles, namely $c_{j}$ and $c_{j+2}$, with the given constraints. This results in a unique pair of bounding circles and thus a unique cyclide piece that joins the previous piece in a tangent-continuous manner. This cyclide piece, in general, will not interpolate the middle circle $c_{j+1}$. However, the circle $c_{j+1}$ can be used to determine which segment of the cyclide to use.

In 3D, only the junction spheres determine the cyclide pieces; the middle spheres act as bias spheres for the selection of the appropriate portion of each cyclide, as shown in Figure 6. This method is described by the following pseudocode:

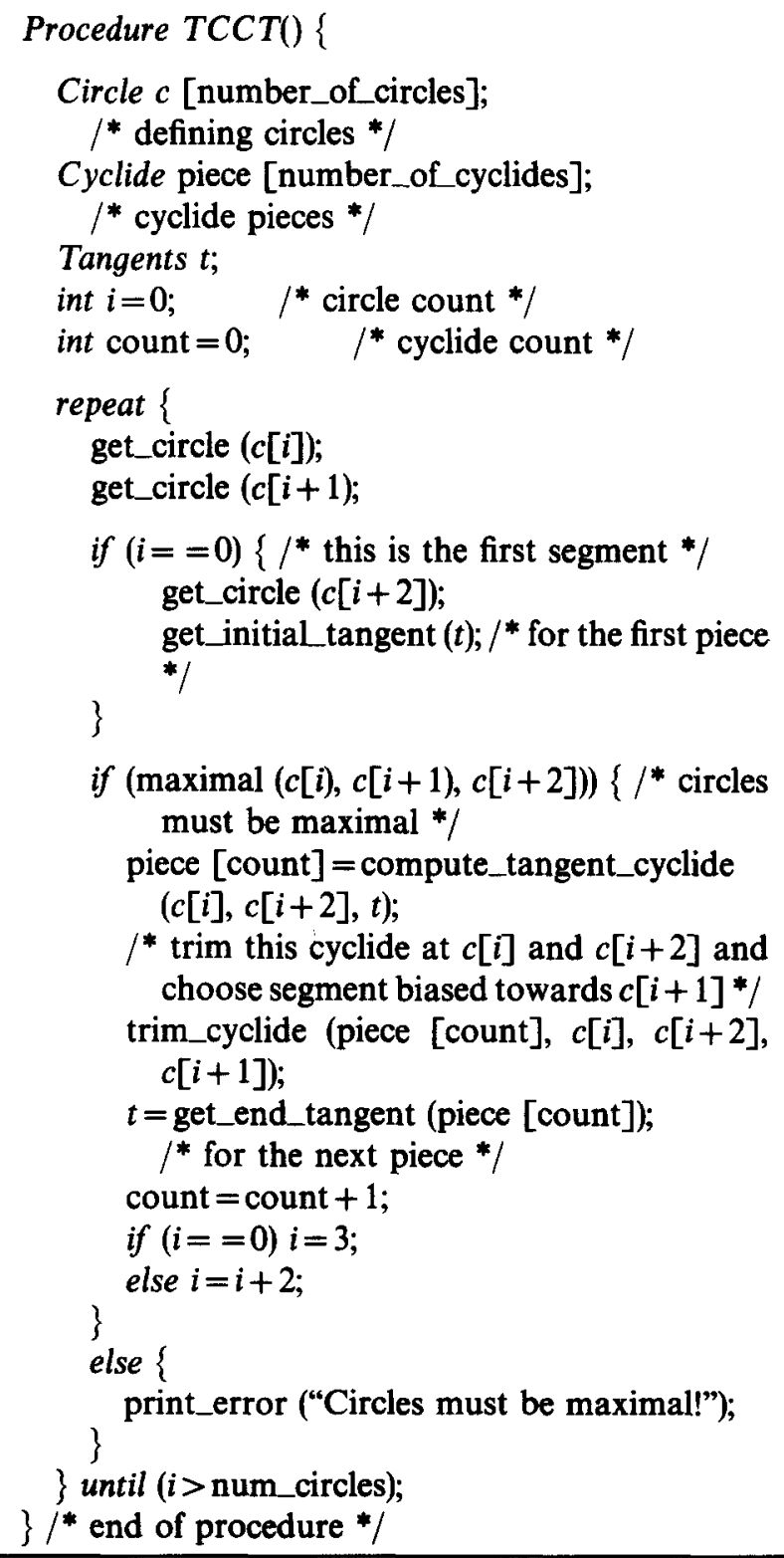

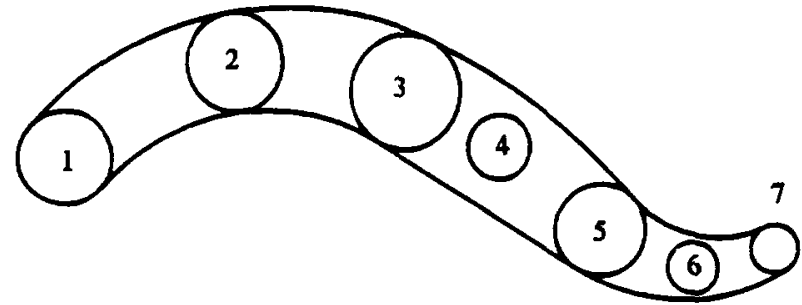

Figure 7 Smoothly joined piecewise cyclide surface generated by TCCT

\section{Comments on TCCT}

We again point out here that the centres of the spheres need not be coplanar. The spine curve of any given segment of a cyclide lies in the plane determined by the axis of the tangent cone at the start of the segment and the centre of the sphere at the end of the segment.

The TCCT ensures that adjacent cyclide pieces join with tangent continuity, without spherical kneecaps. This technique, in comparison with the UCCT, results in a marked improvement in the quality of the freeform objects generated. All the examples shown below are generated using the TCCT. Figure 7 shows a typical construction.

One potential problem with editing objects created using the TCCT arises when the designer alters a junction sphere. Since the TCCT depends on tangent constraints from the previous piece, such a modification produces a cascading effect, resulting in a global change in shape, which is probably not desirable. What we ideally need is an editing method that allows the designer to alter any junction sphere, but that localizes the effect (for example to two or three adjacent pieces). We describe such an editing technique below.

\section{LOCAL EDITING OF COMPOSITE SURFACE}

We present two techniques for achieving local modification of the composite surface. The first method limits the effects of modification to two segments. The second method affects three consecutive segments, but also provides additional flexibility.

\section{Double-cyclide blend editing technique}

The double-cyclide blend editing technique (DCET) is a technique for local modification of the composite surface that is based on the methods for a double-cyclide blend*. It is well known that a family of double-cyclide blends exists for joining two cyclides in space ${ }^{11}$. In fact,

*A double-cyclide blend is a blending technique in which two pieces from (different) cyclides, joined in a tangent-continuous manner, are used to produce a smooth blending surface. Owing to the increased number of degrees of freedom, a double-cyclide blend is more flexible than a single-cyclide blend. 

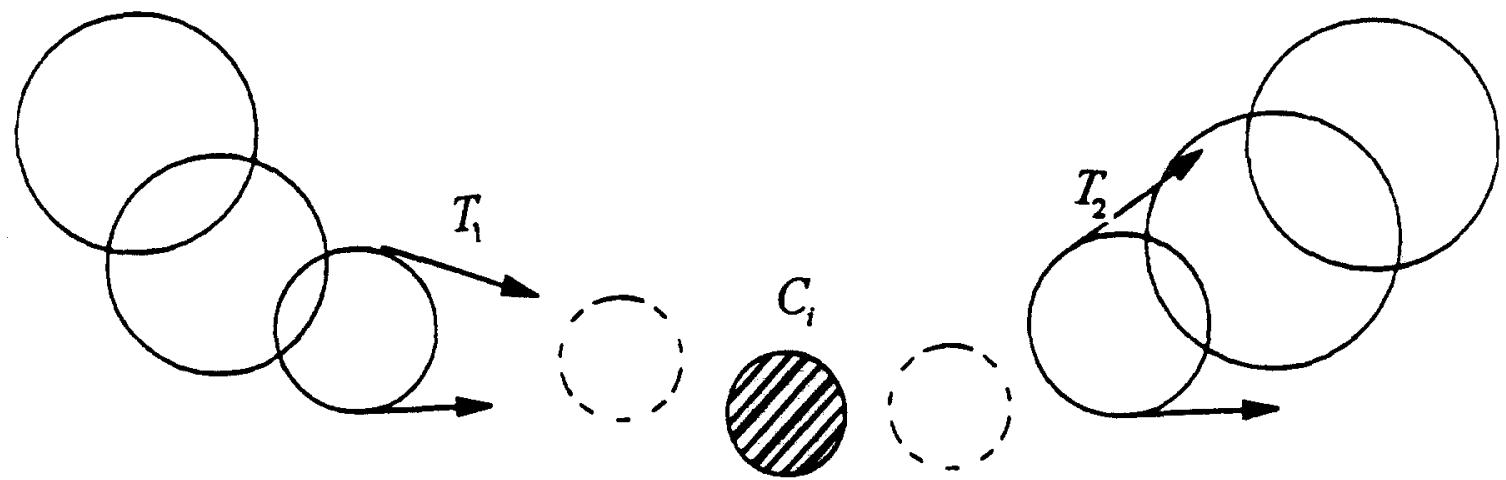

Figure 8 Double-cyclide blend editing
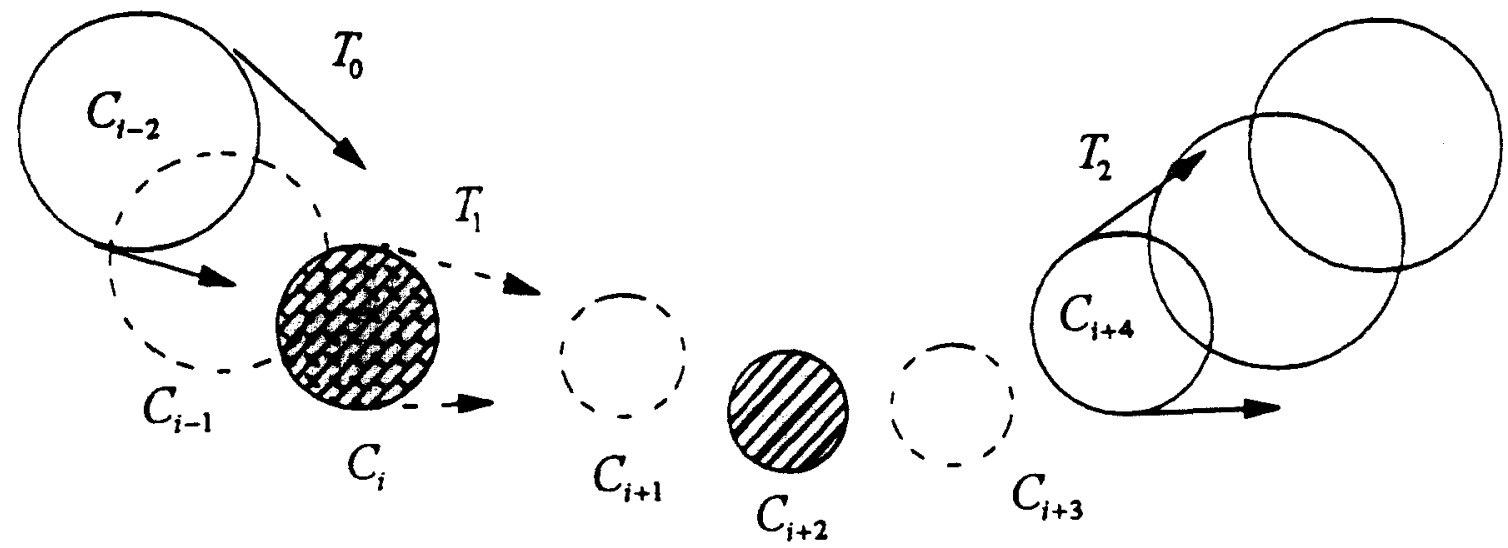

Figure 9 Triple-cyclide blend editing

the cyclide pieces $[i+1]$ and $[i+2]$ belong to a family of double-cyclide blends that join the cyclide pieces [i] and $[i+3]$. By relocating the junction sphere between the cyclide pieces $[i+1]$ and $[i+2]$ appropriately, we can obtain different members of the double-blend family (see Figure 8). In Figure 8, the first three spheres define the piece [i], and hence the tangent cone $T_{1}$ at the end of piece [i]. Similarly, the tangent cone $T_{2}$ at the beginning of the piece $[i+3]$ is fixed. The junction sphere (crosshatched) is labelled as $c_{i}$. Also, the bias spheres for each of the two cyclide segments are shown as broken-line circles. The junction sphere cannot be moved arbitrarily, but is constrained by the biarc requirements as described in Reference 11. Thus, the DCET allows us to restrict the modifications to two pieces at a time, but, because of constraints on the location and size of the junction sphere, it provides only limited flexibility.

\section{Triple-cyclide blend editing technique}

The triple-cyclide blend editing technique (TCET) is a hybrid scheme that combines the advantages of the TCCT composition scheme and the DCET editing method. It provides the ability to alter a junction sphere arbitrarily (see Figure 9). Let us suppose that circle $c_{i}$ (crosshatched) is moved to a new location and possibly resized. Then, we recompute the piece corresponding to $c_{i-2}, c_{i-1}$ and $c_{i}$ (and tangent cone $T_{0}$ ) using the TCCT composition scheme. This procedure fixes the tangent cone $T_{1}$ on sphere $c_{i}$. Next, we compute a double-cyclide blend between $c_{i}$ and $c_{i+4}$ using the DCET method described above. For this step, the junction sphere is $c_{i+2}$ (crosshatched), and the beginning and ending tangent cones are $T_{1}$ and $T_{2}$, respectively. In this process, we obtain another degree of freedom, which can be constrained if it is not required. Thus the change is localized to at most three pieces of the composite surface. The TCET allows a designer to make arbitrary changes to the shape of the composite surface.

\section{EXAMPLES}

In this section, we provide a few examples that illustrate the results of applying the proposed synthesis techniques to geometrically complex shape design. These examples were generated on our native modelling system using the TCCT. It should be pointed out that the circles used for this composition are purely for the purposes of construction. The corresponding spherical surfaces do not contribute to the final composite surface (except when the UCCT is used).

The first example (see Colour Plate 1) shows a crane hook designed using cyclide pieces. In all, 13 spheres and six cyclide segments were used to construct the hook. The 


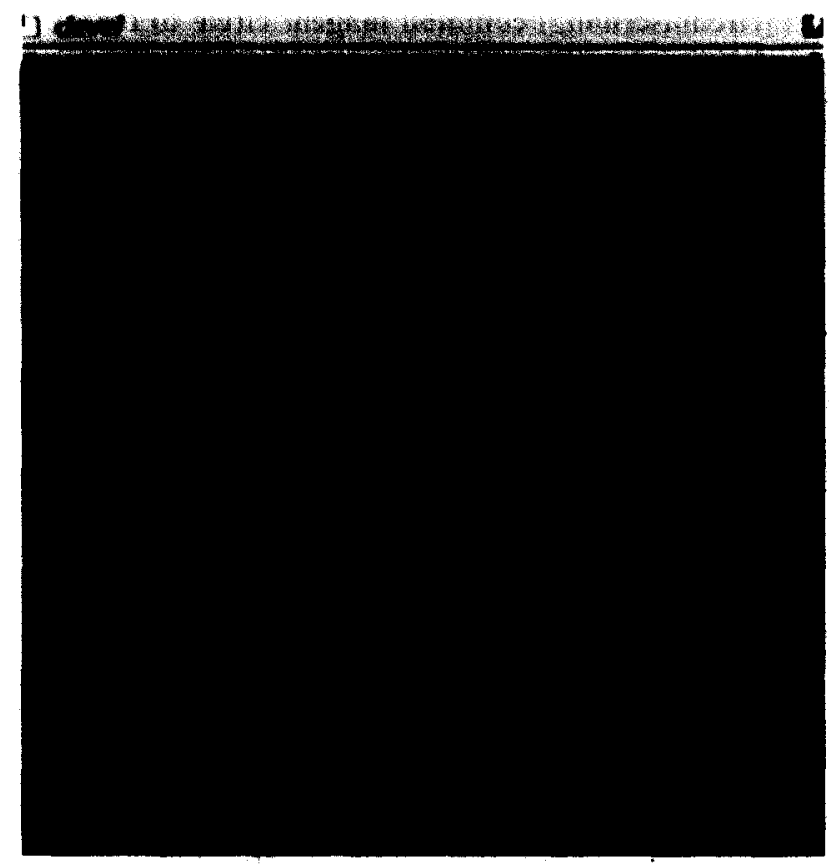

Colour Plate 1 Crane hook designed using TCCT

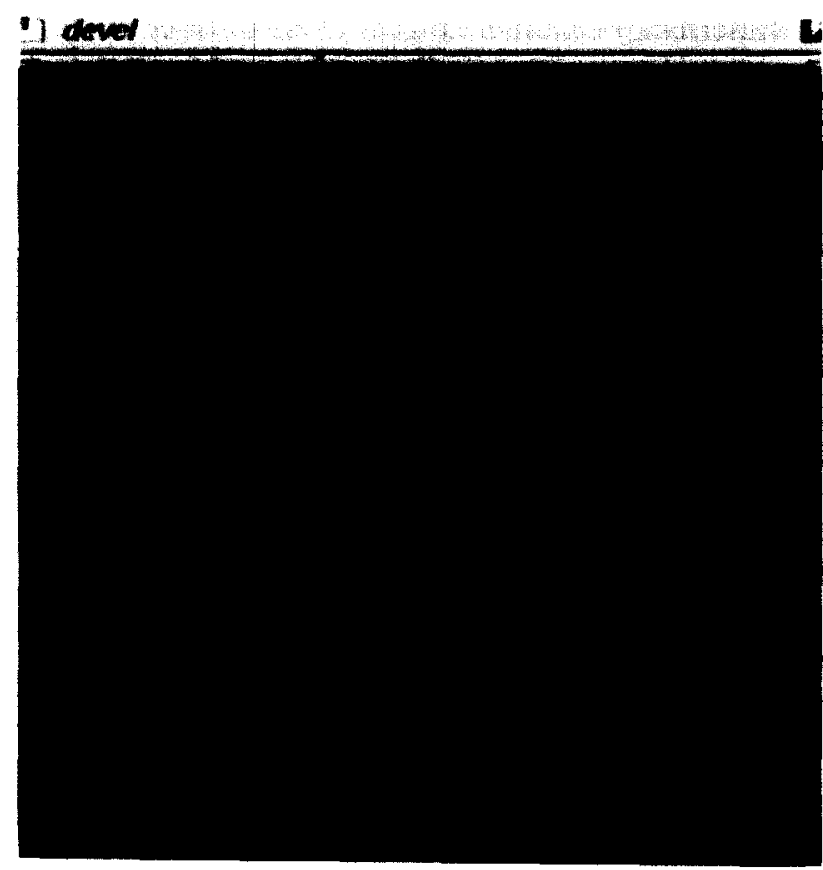

Colour Plate 2 Outer shell of jet engine designed using TCCT

second example (see Colour Plate 2) shows the outer surface of an aircraft jet-engine housing. It was designed using seven spheres, and it is composed of three cyclide segments. The inner housing was then generated by offsetting the outer housing by the thickness of the housing wall. This example uses the property that the offset of a cyclide surface is another cyclide surface. In the third example (see Colour Plate 3), we show an assortment of aesthetic objects generated using the user interface and techniques proposed in this paper.

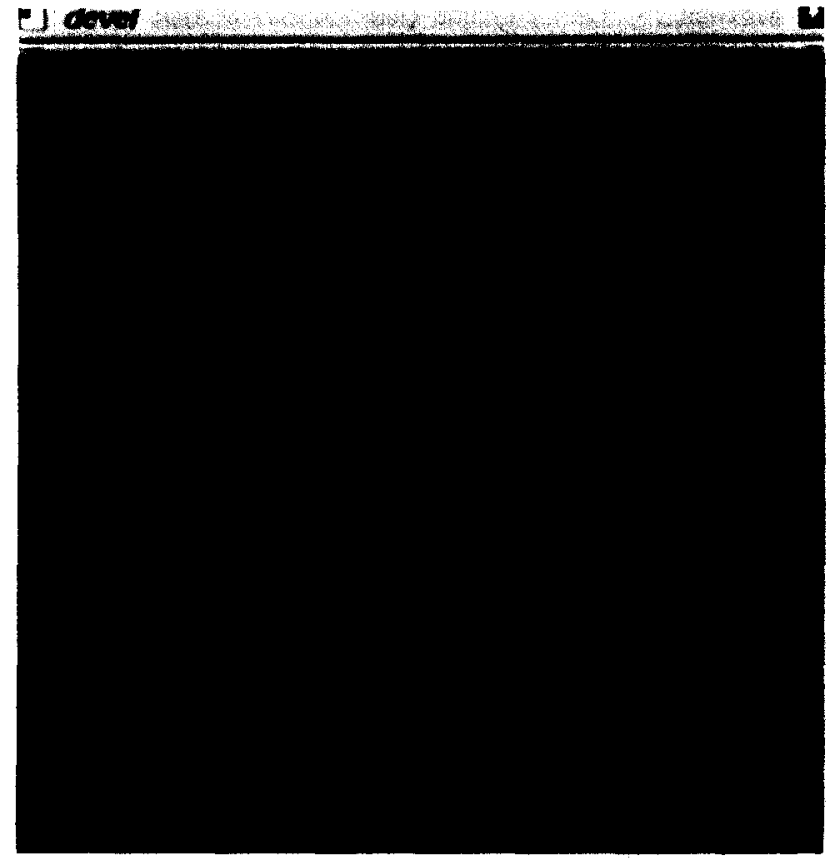

Colour Plate 3 Design of aesthetic shapes using TCCT

Each example was constructed using the TCCT in approximately $2 \mathrm{~min}$.

\section{SUMMARY AND CONCLUSIONS}

We have described a simple, intuitive approach for composing freeform-like objects using tubular cyclide pieces. The designer chooses the spheres that the composite surface will interpolate. The techniques described in this paper generate the composite surface from these defining spheres. The designer can choose to automatically enforce the tangent-continuity requirements on the composite surface. We have also described two editing methods of modifying these composite surfaces locally.

The examples illustrate the effectiveness of this method for the rapid design and modification of practical objects. The potential applications of these methods include diverse areas such as piping layout, wire-harness design and motion planning, as well as the design of mechanical parts.

\section{ACKNOWLEDGEMENTS}

The authors gratefully acknowledge the financial support of US National Science Foundation grant DDM 9010411, and AFOSR grant F49620-93-1-0149.

\section{REFERENCES} 1 Dupin, C Applications de Geometrie et de Mechanique Bachelier,
France (1882) 
2 Maxwell, J C 'On the cyclide' Quart. J. Pure \& Appl. Math. Vol 9 (1868) pp 111-126

3 Cayley, A 'On the cyclide' Quart. J. Pure \& Appl. Math. Vol 12 (1873) p 148

4 Martin, R R 'Principal patches - a new class of surface patch' Proc. Eurographics 1983 North-Holland (1983) pp 111-126

5 Pratt, M J'Applications of cyclide surfaces in geometric modeling' Proc. 3rd IMA Conf. Mathematics of Surfaces Oxford University Press (1988)

6 de Pont, J 'Essays on cyclide patch' PhD Dissertation Darwin College, Cambridge University (1984)

7 Chandru, V, Dutta, D and Hoffmann, C 'Variable radius blending using Dupin cyclides' in Preiss, $\mathrm{K}$, Turner, $\mathrm{J}$ and Wozny, M (Eds.) Geometric Modeling for Product Engineering NorthHolland (1990)

8 Sharrock, T J 'Biarcs in three dimensions' in Martin R R (Ed) The Mathematics of Surfaces II Clarendon Press, UK (1987) (Proc. IMA Conf. Mathematics of Surfaces)

9 McLean, D 'A method for generating surfaces as a composite of cyclide patches' Comput. J. Vol 28 No 4 (1984) pp 433-438

10 Martin, R R, de Pont, $J$ and Sharrock, T $J$ 'Cyclide surfaces in computer aided design' in Gregory, J (Ed.) Mathematics of Surfaces Oxford University Press (1986)

11 Pratt, M J 'Cyclides in geometric design' Comput. Aided Geom. Des. Vol 7 (1990) pp 221-242

12 Srinivas, $Y \mathrm{~L}$ and Dutta, $\mathrm{D}$ 'Motion planning in three dimensions using cyclides' Proc. Computer Graphics Int. Tokyo, Japan (1992) pp 781-791

13 Srinivas, $Y \mathrm{~L}$ and Dutta, $\mathrm{D}$ 'Blending and joining using cyclides' Proc. ASME Design Automation Conf. 1992 Scottsdale, AZ, USA (1992)

14 Chandru, V, Dutta, D and Hoffmann, C 'On the geometry of Dupin cyclides' Visual Comput. Vol 5 (1989) pp 277-290

\section{BIBLIOGRAPHY}

Boehm, W 'On cyclides in geometric modeling' Comput. Aided Geom. Des. Vol 7 (1990) pp 243-255

Hoffmann, C and Hopcroft, J 'Quadratic blending surfaces' Comput.-Aided Des. Vol 18 (1986) pp 301-306

Knapman, J 'Dupin cyclide and the cyclide patch' IBM UK Scientific Centre (1986) (UKSC 156)

Nutbourne, A W and Martin, R R Differential Geometry Applied to the Design of Curves and Surfaces - Vol I Ellis Horwood, UK (1988)

\section{APPENDIX}

In this appendix, we give the mathematical details of the central steps of the UCCT and the TCCT. The core problem is the generation of a circle satisfying one of the following two conditions:

- tangency to three given circles (the UCCT),

- tangency to two circles, and the satisfaction of a point and tangent constraint on one of the circles (the TCCT).

\section{Generation of circle tangent to three maximal circles}

It is well known that three circles in general possess eight different tangent circles. However, in the present work, we are only interested in those tangent circles that either circumscribe or inscribe the given set of circles. This is because we impose the restriction that all of the three spheres that specify the cyclide segment belong to the same family.

\section{Outer (circumscribing) common tangent circle to three circles}

Let the centres and radii of the three circles be (see Figure $10)$ as follows:

- circle 1: centre $(a, b)$, radius $r_{1}$,

- circle 2: centre $(c, d)$, radius $r_{2}$,

- circle 3: centre $(e, f)$, radius $r_{3}$.

Let the centre of the outer tangent circle be $(x, y)$, and the radius be $R$. We can then write the geometric equations describing the problem as follows:

$$
\begin{aligned}
& (x-a)^{2}+(y-b)^{2}=\left(R-r_{1}\right)^{2} \\
& (x-c)^{2}+(y-d)^{2}=\left(R-r_{2}\right)^{2} \\
& (x-e)^{2}+(y-f)^{2}=\left(R-r_{3}\right)^{2}
\end{aligned}
$$

Since we have three equations for three unknowns $(x, y$ and $R$ ), we can proceed to solve for the unknowns. This set of nonlinear equations admits a solution in closed form.

Inner (inscribing) common tangent circle to three circles Here, the problem is very similar to the one posed above. We can write the geometric equations describing the problem as follows:

$$
\begin{aligned}
& (x-a)^{2}+(y-b)^{2}=\left(R+r_{1}\right)^{2} \\
& (x-c)^{2}+(y-d)^{2}=\left(R+r_{2}\right)^{2} \\
& (x-e)^{2}+(y-f)^{2}=\left(R+r_{3}\right)^{2}
\end{aligned}
$$

These equations are solved in a manner similar to that used for the outer tangent circle.

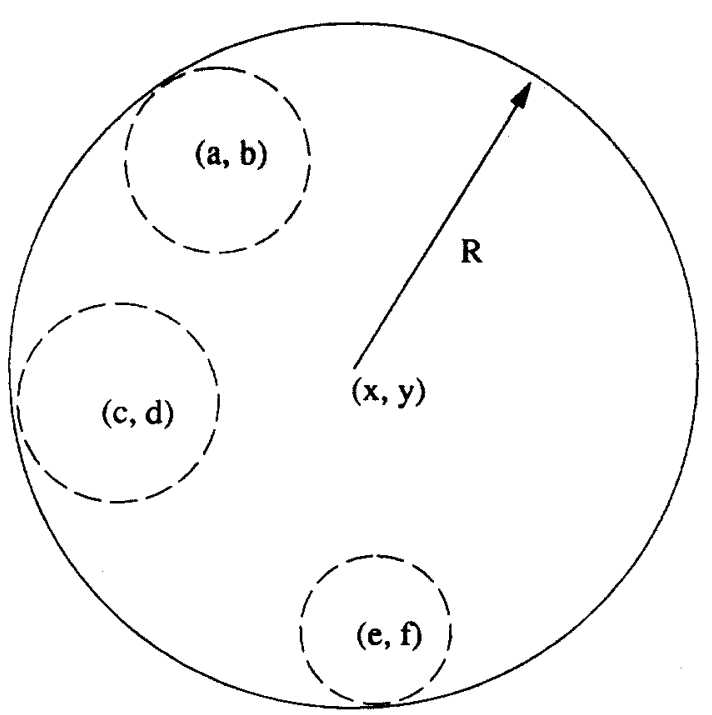

Figure 10 Bounding tangent circle specified by three given circles 


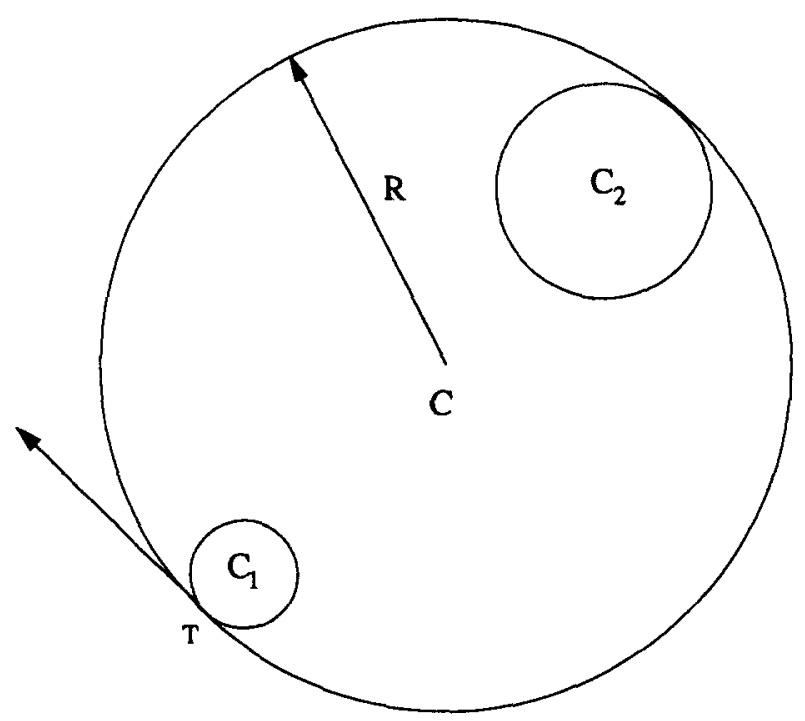

Figure 11 Bounding tangent circle specified by two given circles and one point of tangency

Generation of circle tangent to two maximal circles and satisfying point and tangent constraint on one circle In this case, two solutions exist. However, we are interested in only a circumscribing or an inscribing circle. This additional constraint assures that the solution is unique.

The following quantities are specified (see Figure 11):

- circle 1: centre $C_{1}(a, b)$, radius $r_{1}$,

- circle 2: centre $C_{2}(c, d)$, radius $r_{2}$,

and $T(p, q)$ is a point on circle 1 that the tangent circle must pass through. The tangent direction at $T$ is automatically fixed. We let $C(x, y)$ be the centre of the required tangent circle, and $R$ be its radius.

We can then write the equations describing the situation for obtaining the outer tangent circle as follows:

$$
\begin{aligned}
& |\mathrm{CT}|=R \\
& \mathrm{CT} \cdot \mathrm{CC}_{1}=0 \\
& \left|\mathrm{CC}_{2}\right|=R-r_{2}
\end{aligned}
$$

In Equations 7-9, the bold characters are vectors (e.g. CT is a vector from $C$ to $T$ ). These equations are a set of three nonlinear equations for the three unknowns $(x, y, R)$. Again, this set of equations has a closed-form solution.

Lakshmi Srinivas obtained a bachelor's degree in mechanical engineering from the Indian Institute of Technology, Madras, India, in 1989. and a master's degree in mechanical engineering from the University of Toledo in 1990. He is currently a doctoral student. His research interests include geometric/solid modelling, motion planning, and the blending of surfaces.

Debasish Dutta is an assistant professor. He gained a PhD at Purdue University, USA, in 1989. His areas of interest include geometric modelling, computational geometry in design and manufacturing automation, and descriptive geometry. 